\title{
Correlações entre Características de Carcaça Avaliadas por Ultra-som e Pós-abate em Novilhos Nelore, Alimentados com Altas Proporções de Concentrado
}

\author{
Saulo da Luz e Silva ${ }^{1}$, Paulo Roberto Leme ${ }^{2}$, Angélica Simone Cravo Pereira ${ }^{3}$, \\ Soraia Marques Putrino ${ }^{3}$
}

\begin{abstract}
RESUMO - O objetivo deste trabalho foi estimar correlações entre medidas in vivo obtidas por ultra-som (US) e algumas características de carcaça. Foram utilizados 22 novilhos Nelore, com peso e idade médios de $279 \mathrm{~kg}$ e 24 meses, confinados por 98 dias e alimentados com dietas com elevada proporção de concentrado. O peso vivo, a área olho de lombo (AOLU) e a espessura de gordura subcutânea (EGSU) por US sobre o músculo "Longissimus dorsi" entre a $12^{\mathrm{a}}$ e $13^{\mathrm{a}}$ costelas, foram coletados a cada 28 dias. Foi utilizado um equipamento de US Piemedical Scanner 200 VET, com transdutor linear de $18 \mathrm{~cm}$. Após o abate, foi tomado o peso de carcaça quente (PCQ) e da gordura renal, pélvica e inguinal (GRPI) e calculado o rendimento de carcaça (RC). Após 24 horas de resfriamento, foi medida a área de olho de lombo (AOLC) e a espessura de gordura subcutânea (EGSC) entre a $12^{\mathrm{a}} \mathrm{e} 13^{\mathrm{a}}$ costelas, na carcaça. Tanto a AOLC quanto a EGSC foram subestimadas pelas medidas de US. Foram estimados coeficientes de correlação de Pearson para AOL e EGS medidas na carcaça e por US que foram 0,74 e 0,87 , respectivamente. Foram obtidas também correlações de 0,27 entre RC e AOLU , 0,42 entre RC e AOLC, -0,30 entre RC e EGSU e -0,29 entre RC e EGSC. A GRPI apresentou uma correlação de 0,48 tanto com EGSC quanto com EGSU. Também foi estimada a correlação de Spearman entre AOLU e AOLC e EGSU e EGSC, obtendo-se valores de 0,89 e 0,77, respectivamente.
\end{abstract}

Palavras-chave: área de olho de lombo, avaliação in vivo, bovinos, espessura de gordura subcutânea, Nelore, ultra-sonografia

\section{Correlations Among Carcass Characteristics Taken by Ultrasound and After Slaughter in Nellore Steers Fed High Concentrate Diets}

\begin{abstract}
The objective of this work was to estimate the correlations among measurements taken in vivo with ultrasound equipment with some carcass characteristics measured after slaughter. Twenty two Nellore steers, with average shrunk body weight of $279 \mathrm{~kg}$ and 24 months of age, were fed by 98 days with high concentrate diets. The shrunk body weight, the ribeye area (AOLU) and the fat thickness (EGSU) over the Longissimus dorsi muscle between $12^{\mathrm{a}}$ and $13^{\mathrm{a}}$ ribs, were measured at 28 days intervals. Real-time ultrasound equipment Piemedical Scanner 200 VET, with $18 \mathrm{~cm}$ linear array transducer was utilized. After the slaughter, the hot carcass weight (PCQ) and the kidney, pelvic and inguinal fat (GRPI) were weighted and the dressing percentage (RC) calculated. After 24 hours of cooling the ribeye area (AOLC) and the fat thickness (EGSC) were measured. Both the AOLC and the EGSC were underestimated by ultrasound measurements. The Pearson correlation coefficients for ribeye area and backfat thickness, measured in the carcass and with ultrasound, were 0,74 and 0,87 , respectively. The coefficient between RC and AOLU was 0,27; 0,42 between RC and AOLC, 0,30 between RC and EGSU and $-0,29$ between RC and EGSC. The GRPI presented a 0,48 correlation coefficient with EGSC and EGSU. The Spearman correlation was estimated between AOLU and AOLC and EGSU and EGSC, and the values were 0,89 and 0,77, respectively.
\end{abstract}

Key Words: ribeye area, in vivo evaluation, beef cattle, backfat thickness, Nellore, ultrasound

\section{Introdução}

O conhecimento das características quantitativas e qualitativas de carcaças é fundamental na indústria de carne, visando melhorar a qualidade do produto final. A determinação destas características, de maneira rápida, não invasiva e com boa acurácia, pode ser obtida por ultra-sonografia. Vários pesquisadores empregaram esta técnica para medir características de composição corporal de animais vivos (Stouffer et al., 1961; Kempster \& Owens, 1981; Simm, 1983; Wilson, 1992, entre outros).

Segundo Wilson (1992), a utilização da ultrasonografia para estimar a proporção de músculo e quantidade de gordura é mais acurada do que o peso vivo e outras características facilmente medidas. O

\footnotetext{
${ }^{1}$ Aluno de Doutorado da Faculdade de Zootecnia e Engenharia de Alimentos da Universidade de São Paulo (FZEA/USP). Cx.Postal 23, Pirassununga, SP - CEP: 13.630-000. E.mail: sauloluz@fzea.usp.br

${ }^{2}$ Professor Doutor, Depto. de Zootecnia, FZEA/USP, Pirassununga, SP. E.mail: prleme@usp.br

${ }^{3}$ Aluna de Doutorado da FZEA/USP, Pirassununga, SP.
} 
autor ressalta que há correlação alta e positiva entre porcentagem de gordura subcutânea e porcentagem de gordura de recorte e negativa com porcentagem de carne magra na carcaça.

Hedrick (1983) afirmou que a relação entre a espessura da gordura subcutânea, a área do músculo Longissimus dorsi e a composição da carcaça, medida por ultra-som no animal vivo, tem sido similar às relações entre as mesmas medidas na carcaça. Rouse et al. (1992) também encontraram boas correlações entre as medidas feitas por ultra-som e as feitas posteriormente na carcaça. Entretanto, alguns trabalhos relatam correlações mais baixas entre essas medidas (Ribeiro et al., 1999a, 1999b).

Segundo Perkins (1992), as limitações tecnológicas, a experiência do técnico, o nível de gordura e de músculo, o sexo e a idade do animal, as mudanças nas características dos tecidos pós-morte, a remoção da gordura junto com o couro e o deslocamento dos músculos em relação ao esqueleto são os principais fatores que interferem na acurácia das medidas feitas com o ultra-som e daquelas feitas diretamente na carcaça. Waldner et al. (1992) também afirmaram que diferenças devido a instrumentos, operadores, espessura do couro, comprimento do pêlo, peso e nível de acabamento e corte da carcaça contribuem para essa variação de resultados e induzem alguns pesquisadores a concluírem que alguns equipamentos de ultra-som são insuficientemente acurados e inconsistentes para uso em pesquisa ou na indústria. Entretanto, é importante ressaltar que as diferenças atribuídas a equipamentos são mais im- portantes em se tratando de medidas de gordura intramuscular, com menor influência nas medidas de área de olho de lombo e espessura de gordura subcutânea.

Thwaites (1984) enfatizou que o ultra-som não identifica tecidos ou órgãos, mas simplesmente permite visualizar estruturas sob os tecidos, sendo desta forma necessário que o operador tenha um detalhado conhecimento da anatomia do local em teste e que saiba diferenciar pele, camada de gordura e músculos, entre outros, bem como a variação entre animais. Wilson (1996) também afirmou que um ponto fundamental para o sucesso da utilização desta técnica é uma correta coleta e interpretação das imagens, o que está altamente relacionado ao grau de experiência do técnico.

Dessa forma, o objetivo deste trabalho foi estimar as correlações entre medidas obtidas com o ultrasom e aquelas obtidas diretamente na carcaça, bem como com outras características de interesse econômico e a viabilidade da utilização dessas medidas para determinação das características de carcaça in vivo.

\section{Material e Métodos}

Foram utilizados 22 novilhos Nelore, com peso médio de $279 \mathrm{~kg}$ e 24 meses de idade. Os animais foram confinados por um período de 98 dias, após um período de adaptação de 28 dias, recebendo dietas com 73, 79 ou $85 \%$ de concentrado, sendo o restante da dieta composta por bagaço-de-cana in natura (Tabela 1).

As pesagens foram realizadas no início do período de adaptação, no início do período experimental e

Tabela 1 - Composição percentual das rações, expressos na matéria seca

Table 1 - Percentual composition of diets on a dry matter basis

\begin{tabular}{|c|c|c|c|}
\hline \multirow[t]{2}{*}{$\begin{array}{l}\text { Ingredientes } \\
\text { Ingredients }\end{array}$} & \multicolumn{3}{|c|}{$\begin{array}{c}\text { Níveis de concentrado } \\
\text { Concentrate levels }\end{array}$} \\
\hline & $85 \%$ & $79 \%$ & $73 \%$ \\
\hline Milho grão seco (Corn dry grain) & 41,2 & 37,4 & 33,8 \\
\hline Farelo de soja 49\% (Soybean meal 49\%) & 13,6 & 14,0 & 14,2 \\
\hline Polpa de citrus peletizada (Pelleted citrus pulp) & 28,2 & 25,6 & 23,0 \\
\hline Bagaço de cana in natura (Sugar cane bagasse in natura) & 15,0 & 21,0 & 27,0 \\
\hline Uréia (Urea) & 0,8 & 0,8 & 0,8 \\
\hline Núcleo mineral (Mineral premix) & 1,0 & 1,0 & 1,0 \\
\hline Sulfato de amônia (Amonnium sulphate) & 0,05 & 0,05 & 0,05 \\
\hline Cloreto de potássio (Potassium chloridre) & 0,2 & 0,2 & 0,2 \\
\hline Rumensin (Rumensin) & 0,03 & 0,03 & 0,03 \\
\hline \multicolumn{4}{|l|}{ Nutrientes (Nutrients) 1} \\
\hline Proteína bruta (Crude protein), \% & 14,6 & 14,3 & 14,0 \\
\hline Proteína degradável no rúmen, \% (Remen degradable protein, \%) & 9,9 & 9,8 & 9,6 \\
\hline NDT $(T D N), \%$ & 74,5 & 71,4 & 68,3 \\
\hline
\end{tabular}

${ }^{1}$ Estimado pela fórmula de Weiss et al. (1992) (Estimated by Weiss et al. [1992] formule).

R. Bras. Zootec., v.32, n.5, p.1236-1242, 2003 
a cada 28 dias, sempre após jejum completo de 18 horas, inclusive antes do abate.

Durante as pesagens foram também medidas a área olho de lombo (AOLU) e a espessura de gordura subcutânea (EGSU) por ultra-sonografia, entre a $12^{\mathrm{a}}$ e a $13^{\mathrm{a}}$ costelas, sobre o músculo Longissimus dorsi. As medidas da EGSU e da EGSC foram realizadas a $3 / 4$ da borda medial do músculo Longissimus dorsi. Foi utilizado óleo vegetal como acoplante acústico e um equipamento de ultra-sonografia Piemedical Scanner 200 VET, com transdutor linear de $18 \mathrm{~cm}$, acoplado a uma guia acústica. As imagens obtidas foram gravadas em um computador e analisadas posteriormente.

Ao final do período experimental, os animais foram abatidos no Matadouro Escola da Faculdade de Zootecnia e Engenharia de Alimentos da USP. Durante o abate, foram colhidos o peso de carcaça quente (PCQ), a gordura renal, pélvica e inguinal (GRPI), o peso de carcaça quente (PCQ) e calculado o rendimento de carcaça (RC). Após 24 horas de resfriamento em câmara fria a $2^{\circ} \mathrm{C}$, as carcaças foram novamente pesadas, obtendose o peso da carcaça fria (PCF). As meia-carcaças esquerdas foram seccionadas na região entre a $12^{\mathrm{a}}$ e a $13^{\mathrm{a}}$ costelas e medidas com o auxílio de uma régua especial a área de olho de lombo (AOLC) e a espessura de gordura subcutânea (EGSC) na carcaça.

Foram estimadas correlações de Pearson e Spearman para determinar a associação entre as medidas feitas por ultra-som e aquelas tomadas diretamente na carcaça. Os tratamentos (níveis de concentrado) não foram considerados na análise estatística para o cálculo das correlações e regressões.

Além dos dados de ultra-som e da carcaça, foram criadas duas variáveis contendo as diferenças atuais entre as medidas feitas por ultra-som e na carcaça. As variáveis criadas foram:

$$
\begin{aligned}
& \text { DIFEGS }=\text { EGSU - EGSC; } \\
& \text { DIFAOL = AOLU - AOLC; }
\end{aligned}
$$

em que: DIFEGS = diferença entre a EGSU e a EGSC; DIFAOL $=$ diferença entre a AOLU e a AOLC;

Essas diferenças indicam a direção da tendência no momento da interpretação das medidas por ultrasom e na carcaça.

As análises estatísticas foram realizadas utilizando-se os procedimentos REG e COR do programa SAS (2001).

\section{Resultados e Discussão}

Na Tabela 2, estão as médias, erros-padrão, mínimos e máximos dos dados analisados das características nos animais vivos e pós-abate.

Tabela 2 - Médias e erros-padrão (EP) das medidas in vivo e na carcaça

\begin{tabular}{|c|c|c|c|c|}
\hline $\begin{array}{l}\text { Características } \\
\text { Traits }\end{array}$ & $\begin{array}{c}\text { Média } \\
\text { Mean }\end{array}$ & $\begin{array}{l}\mathrm{EP} \\
S E\end{array}$ & $\begin{array}{l}\text { Mínimo } \\
\text { Minimum }\end{array}$ & $\begin{array}{l}\text { Máximo } \\
\text { Maximum }\end{array}$ \\
\hline $\begin{array}{l}\text { Peso vivo final, } \mathrm{kg} \\
\text { Final body weight, } \mathrm{kg}\end{array}$ & 424 & 6,24 & 375 & 493 \\
\hline $\begin{array}{l}\text { Ganho médio diário, } \mathrm{kg} \\
\text { Average daily gain, } \mathrm{kg}\end{array}$ & 1,48 & 0,03 & 1,06 & 1,79 \\
\hline $\begin{array}{l}\text { Peso carcaça quente, } \mathrm{kg} \\
\text { Hot carcass weight, } \mathrm{kg}\end{array}$ & 244,9 & 3,48 & 208,6 & 272,0 \\
\hline $\begin{array}{l}\text { Rendimento de carcaça, } \% \\
\text { Dressing percentage, } \%\end{array}$ & 57,7 & 0,30 & 55,2 & 60,6 \\
\hline $\begin{array}{l}\text { Gordura renal, pélvica e inguinal, } \mathrm{kg} \\
\text { Kidney, pelvic and inguinal fat, } \mathrm{kg}\end{array}$ & 8,4 & 0,36 & 6,0 & 12,0 \\
\hline $\begin{array}{l}\text { Área de olho de lombo - ultra-som, } \mathrm{cm}^{2} \\
\text { Ultrasound ribeye area, } \mathrm{cm}^{2}\end{array}$ & 57,4 & 1,05 & 46,37 & 66,79 \\
\hline $\begin{array}{l}\text { Espessura de gordura - ultra-som, mm } \\
\text { Ultrasound backfat tickness, } \mathrm{mm}\end{array}$ & 7,4 & 0,60 & 3,1 & 13,5 \\
\hline $\begin{array}{l}\text { Área de olho de lombo - carcaça, } \mathrm{cm}^{2} \\
\text { Carcass ribeye area, } \mathrm{cm}^{2}\end{array}$ & 60,5 & 1,30 & 50,0 & 72,0 \\
\hline $\begin{array}{l}\text { Espessura de gordura - carcaça, mm } \\
\text { Carcass backfat tickness, mm }\end{array}$ & 8,5 & 0,93 & 2,0 & 17,0 \\
\hline $\begin{array}{l}\text { DIFEGS,mm } \\
D F A T, m m\end{array}$ & $-1,05$ & 0,50 & $-6,1$ & 3,2 \\
\hline $\begin{array}{l}\text { DIFAOL, } \mathrm{cm}^{2} \\
D A O L, \mathrm{~cm}^{2}\end{array}$ & $-3,1$ & 0,88 & $-8,86$ & 6,8 \\
\hline
\end{tabular}

Table 2 - Means and standard errors (SE) of measures in vivo and in the carcass

DIFEGS - Diferença entre EGSU e EGSC, DIFAOL - Diferença entre AOLU e AOLC.

DFAT - Ultrasound backfat thickness minus carcass backfat thickness, DREA - Ultrasound ribeye area minus carcass ribeye area.

R. Bras. Zootec., v.32, n.5, p.1236-1242, 2003 
No momento do abate, os animais apresentaram peso vivo médio de $424 \mathrm{~kg}$, com ganho médio diário de $1,480 \mathrm{~kg}$ durante o período experimental. A média de PCQ foi de $244,9 \mathrm{~kg}$, com um RC médio de $57,7 \%$. A EGSU e EGSC foram de 7,4 e $8,5 \mathrm{~mm}$ e a AOLU e AOLC 57,4 e $60,5 \mathrm{~cm}^{2}$, respectivamente.

A DIFEGS apresentou valor médio de $-1,05 \mathrm{~mm}$, indicando que houve tendência de subestimação da EGS. Tendência similar (média $-1,7 \mathrm{~mm}$ ) foi obtida por Hassen et al. (1998), trabalhando com animais com média de EGSC de $11 \mathrm{~mm}$. Entretanto, segundo os autores a gordura na carcaça foi superestimada em animais com EGSC inferior a $6 \mathrm{~mm}$ e subestimadas em animais com EGSC superior a $8 \mathrm{~mm}$, sendo que essa diferença aumentou com o maior grau de acabamento dos animais. Esses dados também estão de acordo com os obtidos por Waldner et al. (1992), que avaliaram as relações entre medidas de ultrasonografia e na carcaça em tourinhos Brangus dos 4 aos 24 meses, com EGS média de 5,1 mm aos 12 meses, aumentando progressivamente até atingirem média de 19,3 mm aos 24 meses. Os autores verificaram que à medida que a EGSC aumentava havia tendência de maior subestimação da mesma, partindo de $-0,8 \mathrm{~mm}$ aos 12 meses até $-6,6 \mathrm{~mm}$ aos 24 meses.

Já a DIFAOL teve média de $-3,1 \mathrm{~cm}^{2}$, indicando que a maior parte das medidas de AOLU também foi subestimada. Waldner et al. (1992) relataram que as medidas por ultra-som subestimaram animais com AOLC menores que $70 \mathrm{~cm}^{2}$ e superestimaram de animais com mais de $85 \mathrm{~cm}^{2}$. Já Hassen et al. (1998)

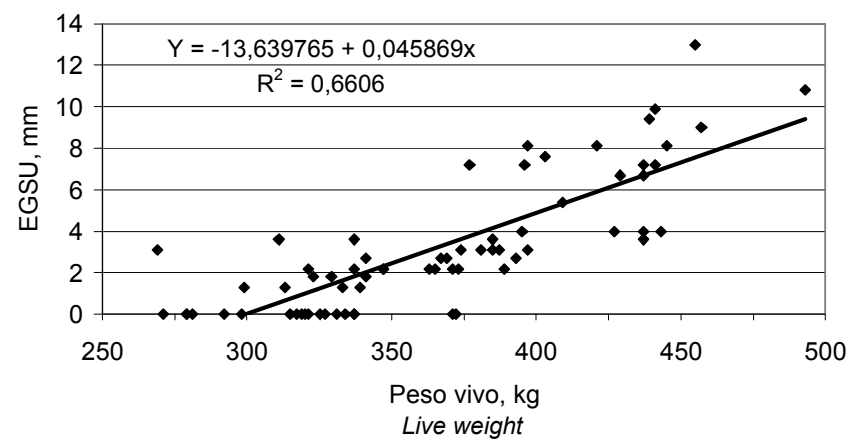

Figura 1 - Dispersão da espessura de gordura subcutânea medida por ultra-som (EGSU) em função do peso vivo.

Figure 1 - Ultrasound backfat thickness (EGSU) as function of body weight.

R. Bras. Zootec., v.32, n.5, p.1236-1242, 2003 encontraram valores de $0,63 \mathrm{~cm}^{2}$, bastante inferiores aos obtidos neste trabalho. Os resultados obtidos neste trabalho estão de acordo com os dados da literatura que indicam subestimação da AOLC em animais com menos que $70 \mathrm{~cm}^{2}$ de AOL.

Com base nesses resultados, é possível sugerir que as medidas de ultra-sonografia para determinar AOLU e EGSU devem ser realizadas em animais com AOLC entre 70 e $85 \mathrm{~cm}^{2}$, enquanto a medida de EGSC deve ser realizada quando os animais possuírem entre 3 e $6 \mathrm{~mm}$, para que se possa obter maior acurácia dessas medidas.

Nas Figuras 1 e 2, está o comportamento da espessura de gordura subcutânea e área de olho de lombo, medidas por ultra-som, ao longo do período experimental. Pode-se observar que tanto a EGSU quanto AOLU apresentaram crescimento linear $(p<0,01)$ em função do peso. De acordo com a Figura 1 , observa-se que até $350 \mathrm{~kg}$ de peso vivo, houve grande número de animais com EGSU igual a zero e a partir desse peso a gordura começou a ser depositada, de forma mais acentuada, aumentando esse ímpeto de crescimento ao redor dos $400 \mathrm{~kg}$.

A área de olho de lombo apresentou um crescimento linear em relação ao peso vivo $(p<0,01)$. Silva (2002) e Nash (2000) também descreveram comportamento semelhante.

Foram estimados os coeficientes de correlação de Pearson para as características analisadas e os resultados estão apresentados na Tabela 3 . O peso vivo final (PVF) foi altamente correlacionado $(p<0,01)$

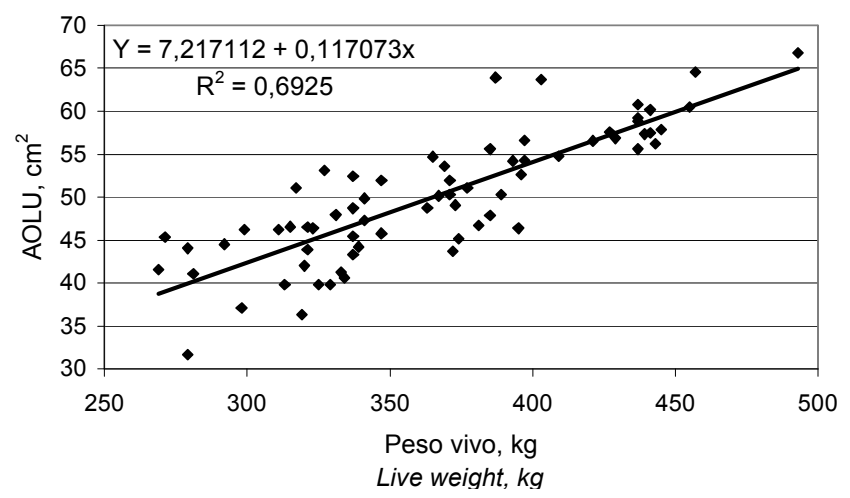

Figura 2 - Dispersão da área de olho de lombo medida por ultra-som (AOLU) em função do peso vivo.

Figure 2 - Ultrasound ribeye area (AOLU) as function of body weight. 
com peso de carcaça quente $(r=0,94)$. Waldner et al. (1992) encontraram correlação de 0,91 entre PV e PCQ. Estes resultados estão de acordo com a literatura que tem reportado altas correlações entre o PV e PCQ (Waldner et al., 1992; Hassen et al., 1999; Silva, 2002).

A EGSU e a AOLU foram altamente $(\mathrm{p}<0,01)$ correlacionadas com EGSC e AOLC ( $\mathrm{r}=0,87$ e $\mathrm{r}=0,74$, respectivamente). Waldner et al. (1992), obteve correlações semelhantes ( $\mathrm{r}=0,86$ e $\mathrm{r}=0,73$, respectivamente), aos obtidos neste trabalho. Já Ribeiro et al. (1999a), Ribeiro et al. (1999b) e Hassen et al. (1998) relataram correlações menores. Rouse et al. (1992), encontraram correlações de 0,91 e 0,86 para EGS e AOL medidas por ultra-som e na carcaça, respectivamente.

Smith et al. (1992), em dois experimentos, testaram a eficiência da ultra-sonografia para estimar AOL e EGS de 452 novilhos confinados. Em ambos os experimentos, as correlações entre EGSU e EGSC foram de 0,81 e 0,82 , respectivamente. Já entre AOLU e AOLC (medida através de grade específica para esse fim), obtiveram correlações de 0,43 e 0,63 , respectivamente. Essa menor correlação se deve ao fato que a AOLU foi obtida com um transdutor menor do que os utilizados atualmente para a avaliação de carcaças. Esse tipo de transdutor, devido ao seu tamanho não permite que toda a área do músculo Longissimus dorsi seja obtida de uma única vez e exige a utilização de um recurso dos equipamentos de ultra-som, que divide a tela em duas partes. Dessa forma, obtém-se a imagem de uma metade do músculo e, em seguida, tenta-se "encaixar" a outra metade do músculo através da observação da imagem na tela do aparelho. Esse tipo de técnica induz a um maior erro na estimativa da AOLU, o que poderia explicar as baixas correlações obtidas. Nesse mesmo trabalho, foram comparadas as medidas realizadas através da grade especial para medida da AOLC e através do delineamento manual do músculo em papel acetato e a correlação obtida foi de 0,89 , demonstrando que as medidas realizadas diretamente na carcaça por métodos diferentes, não são exatamente iguais.

Em relação às demais características, o RC apresentou correlações de 0,27 e 0,42 com AOLU e AOLC e -0,30 e -0,29 com EGSU e EGSC respectivamente. Já a característica GRPI apresentou correlação de 0,48 tanto para EGSU quanto para EGSC. Waldner et al. (1992) encontraram correlação de 0,47 e 0,15 entre AOLU e EGSU com GRPI. Os mesmos autores encontraram correlação de 0,22 e 0,58 entre EGSU e AOLU com peso de carcaça quente, semelhantes aos 0,36 e 0,73 encontrados neste trabalho.

As correlações obtidas pelo Teste de Spearman mostraram que a AOLU teve correlação de 0,77 com a AOLC, enquanto a EGSU apresentou correlação de 0,89 com EGSC. Hassen et al. (1998), relataram coeficientes de correlação de Spearman de 0,71 entre EGSU e EGSC e 0,44 para AOLU e AOLC. Esses valores indicam que as medidas realizadas por ultra-som ou na carcaça, não alteraram a classificação relativa dos animais.

Uma explicação para os maiores valores obtidos neste trabalho é que a coleta das imagens, interpretação e medidas na carcaça foram feitas pelo mesmo técnico, diferente do trabalho de Hassen et al.(1998), onde a coleta e a interpretação das imagens foram realizadas por técnicos diferentes.

Tabela 3 - Coeficientes de correlação de Pearson entre medidas realizadas por ultra-som e na carcaça Table 3 - Coefficients of Pearson correlation between measures taken by ultrasound and on the carcass

\begin{tabular}{|c|c|c|c|c|c|c|c|c|c|}
\hline $\begin{array}{l}\text { Características } \\
\text { Traits } \\
\end{array}$ & $\begin{array}{l}\text { PVF } \\
F B W\end{array}$ & $\begin{array}{l}\text { GMD } \\
A D G \\
\end{array}$ & $\begin{array}{l}\mathrm{PCQ} \\
\mathrm{HCW} \\
\end{array}$ & $\begin{array}{l}\mathrm{RC} \\
D P\end{array}$ & $\begin{array}{l}\text { GRPI } \\
K P I F \\
\end{array}$ & $\begin{array}{c}\text { EGSC } \\
C F T\end{array}$ & $\begin{array}{c}\text { EGSU } \\
U F T \\
\end{array}$ & $\begin{array}{r}\text { AOLC } \\
C R E A \\
\end{array}$ & $\begin{array}{l}\text { AOLU } \\
\text { UREA } \\
\end{array}$ \\
\hline$P V F(F B W), \mathrm{kg}$ & 1,0 & 0,72 & 0,94 & $-0,24$ & 0,22 & 0,34 & 0,22 & 0,30 & 0,63 \\
\hline$G M D(A D G), k g$ & & 1,0 & 0,71 & $-0,09$ & 0,29 & 0,32 & 0,28 & 0,44 & 0,60 \\
\hline PCQ $(H C W), \mathrm{kg}$ & & & 1,0 & 0,11 & 0,22 & 0,25 & 0,12 & 0,45 & 0,73 \\
\hline $\mathrm{RC}(D P), \%$ & & & & 1,0 & $-0,04$ & $-0,29$ & $-0,30$ & 0,42 & 0,28 \\
\hline GRP $(K P F), \mathrm{kg}$ & & & & & 1,0 & 0,48 & 0,48 & $-0,14$ & $-0,08$ \\
\hline $\mathrm{EGSC}(C F T), \mathrm{mm}$ & & & & & & 1,0 & 0,87 & 0,08 & 0,22 \\
\hline $\mathrm{EGSU}(U F T), \mathrm{mm}$ & & & & & & & 1,0 & 0,00 & 0,17 \\
\hline $\operatorname{AOLC}(C R E A), \mathrm{cm}^{2}$ & & & & & & & & 1,0 & 0,74 \\
\hline $\operatorname{AOLU}(U R E A), \mathrm{cm}^{2}$ & & & & & & & & & 1,0 \\
\hline
\end{tabular}

PVF - peso vivo final; GMD - ganho médio diário; PCQ - peso de carcaça quente, RC - rendimento de carcaça; GRP - gordura renal e pélvica; EGSC - espessura de gordura subcutânea na carcaça; EGSU - espessura de gordura subcutânea por ultra-som; AOLC área de olho de lombo na carcaça; AOLU - área de olho de lombo por ultra-som.

FBW - final body weight; $A D G$ - average daily gain; HCW - hot carcass weight; DP - dressing percentage; KPF - kidney and pelvic fat; CFT - carcass fat thickness; UFT - ultrasound fat thickness; CREA - carcass ribeye area; UREA - ultrasound ribeye area. 
A Figura 3 mostra a dispersão dos dados de EGSC em função da EGSU. A regressão da AOLC, em função da AOLU, apresentou $\mathrm{R}^{2}$ de 0,76 .

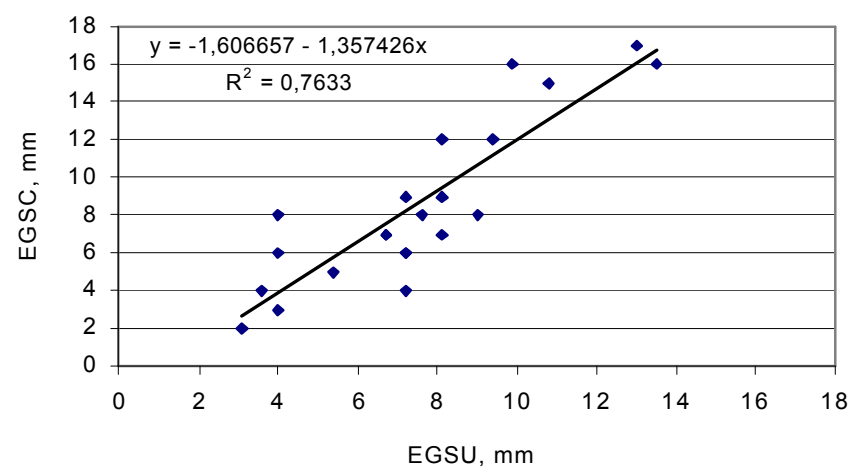

Figura 3 - Espessura de gordura subcutânea medida na carcaça (EGSC), em função da espessura de gordura subcutânea medida por ultra-som (EGSU).

Figure 3 - Carcass backfat thickness (CBFT), as function of ultrasound backfat thickness (EGSU).

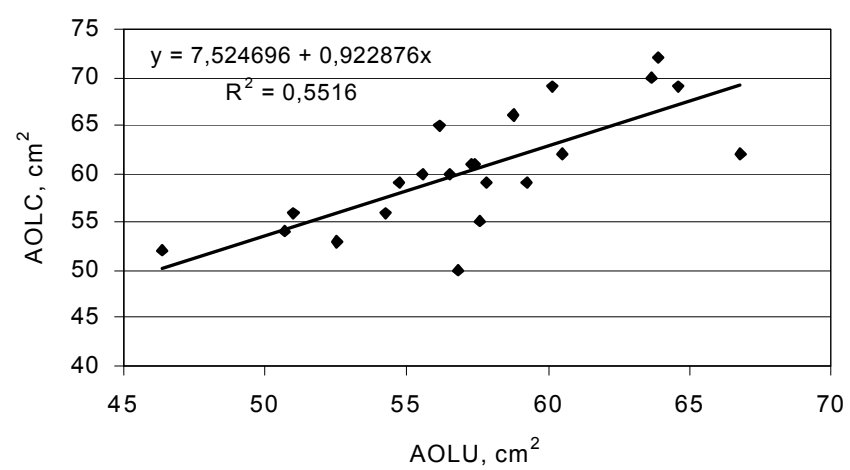

Figura 4 - Área de olho de lombo medida na carcaça (AOLC) em função da área de olho de lombo medida por ultra-som (AOLU).

Figure 4 - Carcass ribeye area (CREA) as function of ultrasound ribeye area (UREA).

\section{Conclusões}

Medidas de área de olho de lombo e espessura de gordura subcutânea feitas por ultra-sonografia apresentaram menores valores em relação às medidas correspondentes na carcaça.

O ultra-som mostrou-se acurado para estimar a área de olho de lombo no momento do abate $\mathrm{e}$ também para classificar os animais.

\section{Literatura Citada}

HASSEN, A.; WILSON, D.E.; WILLHAM, R.L. et al. Evaluation of ultrasound measurements of fat thickness and longissimus muscle area in feedlot cattle: Assessment of accuracy and repeatibility. Canadian Journal of Animal Science, v.78, n.3, p.277-285, 1998.

HASSEN, A.; WILSON, D.E.; ROUSE, G.H. Evaluation of carcass, live, and real-time ultrasound measures in feedlot cattle: II Effect of different age end points on the accuracy of predicting the percentage of retail product, retail product weight, and hot carcass weight. Journal of Animal Science, v.77, p.283-290, 1999.

HEDRICK, H.B.Methods of estimating live animal and carcass composition. Journal of Animal Science, v.57, n.5, p.13161326, 1983.

KEMPSTER, A.J.; OWEN, M.G. A note of the accuracy of an ultrasonic technique for selecting cattle of different breeds for slaughter at equal fatness. Animal Production, v.32, p.113-115, 1981.

SILVA, S.L. Estimativa de características de carcaça e ponto ideal de abate por ulta-sonografia, em bovinos submetidos a diferentes níveis energéticos na ração. Pirassununga: Faculdade de Zootecnia e Engenharia de Alimentos, 2002, 65p. Dissertação (Mestrado em Zootecnia) - Faculdade de Zootecnia e Engenharia de Alimentos/Universidade de São Paulo, 2002.

NASH, S.A.; HARRISON, S.N.; PACKHAM, J.H. et al. Case study: Monitoring changes in carcass quality across time-onfeed using real-time ultrasound to optimize marketing endpoints. The Professional Animal Scientist, n.16, p.202-205, 2000.

PERKINS, T.L. The use of real-time, linear-array ultrasound techniques to predict final carcass composition in beef catle. Ames: Texas Tech University, 1992. Tese (PhD) Texas Tech University, 1992.

RIBEIRO, F.G., LEME, P.R., HENRIQUE, W. et al. Correlações entre medidas com ultra-som "in vivo" e diretamente na carcaça "post mortem" em bovinos. In: CONGRESSO DE ZOOTECNIA, 9., 1999, Portugal. Anais... Portugal: APEZ, 1999a. p.19.

RIBEIRO, F.G., BULLE, M.L.M., LEME, P.R. et al. Relação entre espessura de gordura e área de olho de lombo determinados in vivo e post mortem em bovinos jovens. In: REUNIÃO ANUAL DA SOCIEDADE BRASILEIRA DE ZOOTECNIA, 36., 1999, Porto Alegre. Anais... Porto Alegre: Sociedade Brasileira de Zootecnia, 1999b. p.357.

ROUSE, G.H.; WILSON, D.E.; DUELLO D.A. et al. The accuracy of real-time ultrasound scans taken serially on small-, medium, and large-frame steers and bulls slaughtered at three endpoints. Beef \& Sheep Research Report. Ames: Iowa State University, 1992. p.14-19.

SAS INSTITUTE. SAS/STAT. User's Guide, Version 6, 4 ed., v..2, Cary, North Carolina: SAS Institute Inc., 2001.

SIMM, G. The use of ultrasound to predict the carcass composition of live cattle - a Review. Animal Breeding Abstracts, v.58, n.12, p.853-875, 1983.

SMITH, M.T.; OLTJEN, J.W.; DOLEZAL, H.G. et al. Evaluation of ultrasound for prediction of carcass fat thickness and longissimus muscle area in feedlot steers. Journal of Animal Science, v.70, p.29-37, 1992.

STOUFFER, J.R., WALLENTINE, M.V., WELLINGTON, G.A. Development and application of ultrasonic methods for 
measuring fat thickness and rib-eye area in cattle and rib-eye in cattle and hogs. Journal of Animal Science, v.18, n.4, p.759-767, 1961.

THWAITES, C.J. Ultrasonic estimation of carcass composition - Review. Australian Meet Research Committee, n. 47, 1984. 29p.

WALDNER, D.N.; DIKEMAN, M.E.; SCHALLES, R.R. et al. Validation of real-time ultrasound technology for predicting fat thicknesses, longissimus muscle areas, and composition of Brangus bulls from 4 months to 2 years of age. Journal of Animal Science, v.70, p.3044-3054, 1992.

WEISS, W.P.; CONRAD, H.R.; PIERRE, N.R.St. A theoreticalbased model for predicting total digestible nutrient values of forages and concentrates. Animal Feed Science and Technology, v.39, p.95-110, 1992.
WILSON, D.E. Aplication of ultrasound for genetic improvement. Journal of Animal Science, v.70, p.973-983, 1992.

WILSON, D.E. Improving beef cattle genetics using ultrasound. In: CONGRESSO INTERNACIONAL DE ZootecniA, 1., 1996, Porto Alegre. Anais... Porto Alegre, 1996. p.201.

Recebido em: 05/08/02

Aceito em: 09/01/03 